\title{
Coupling Current and Hysteresis Losses of Bi2212 Round Wires with Twisted Filaments
}

Yifeng Yang, J. Pelegrin, EA Young, I. Falorio,

Institute of Cryogenics, University of Southampton, UK

H. Miao, Y. Huang

Oxford Superconducting Technology, Carteret, NJ, USA

Amalia Ballarino, Luca Bottura

CERN, Geneva, Switzerland

Keywords: Superconducting Magnets, High Temperature Superconductors

\begin{abstract}
AC loss measurements have been carried out on Bi2212 round wires of different twist pitches. With systematical variation of sample temperature $(5 \mathrm{~K}-70 \mathrm{~K})$ and ac field amplitude $(\leq 0.2 \mathrm{~T})$ as well as frequency ( $20 \mathrm{~Hz}-2 \mathrm{kHz}$ ), this work shows (a) the full coupling of the non-twisted wire, (b) the uncoupling of sub-wires by twisting at moderate pitch up to $12 \mathrm{~mm}$, (c) a relatively short time constant of milliseconds for the coupling current due to a high transverse resistivity, and (d) an effective (de)coupling diameter of $0.4 \mathrm{~mm}$ about $1.6 \mathrm{x}$ of sub-wire bundles. The results suggest a promising potential for improving filament decoupling.
\end{abstract}

Presented at: ASC 2014, 10-15 August, Charlotte, USA

Geneva, Switzerland

February 2015 


\title{
Coupling Current and Hysteresis Losses of Bi2212 Round Wires with Twisted Filaments
}

\author{
Yifeng Yang, J Pelegrin, E A Young, I Falorio, A Ballarino, L Bottura, H Miao, Y Huang
}

\begin{abstract}
AC loss measurements have been carried out on Bi2212 round wires of different twist pitches. With systematical variation of sample temperature $(5 \mathrm{~K}-70 \mathrm{~K})$ and ac field amplitude $(\leq 0.2 \mathrm{~T})$ as well as frequency $(20 \mathrm{~Hz}-2 \mathrm{kHz})$, this work shows (a) the full coupling of the non-twisted wire, (b) the uncoupling of sub-wires by twisting at moderate pitch up to 12 $\mathrm{mm}$, (c) a relatively short time constant of milliseconds for the coupling current due to a high transverse resistivity, and (d) an effective (de)coupling diameter of $0.4 \mathrm{~mm}$ about $1.6 \mathrm{x}$ of sub-wire bundles. The results suggest a promising potential for improving filament decoupling.
\end{abstract}

Index Terms-Superconducting Magnets, High Temperature Superconductors.

\section{INTRODUCTION}

$\mathrm{B}$ I2212 ROUND wire has become an exciting prospect [1][3] for high field magnets above $25 \mathrm{~T}$, going beyond the practical limit of start-of-the-art $\mathrm{Nb}_{3} \mathrm{Sn}$ conductors. The performance of Bi2212 conductors has seen a phenomenal increase as the results of high pressure processing [4] and coordinated material research. The round wire configuration of Bi2212 brings substantial advantages over other high temperature superconducting (HTS) tapes, especially in terms of coil manufacturing and filament refinement for large numbers and small sizes. To fully utilize the benefit of small filament size for field quality and cryogenic stabilization, filament twisting is a crucial technical prerequisite. The main challenges are the minimization of critical current degradation and a sufficiently high transverse resistivity to ensure consistent filament uncoupling over long lengths. The fabrication of Bi2212 wires with twisted filaments with clear indications of successful filament uncoupling has been reported recently by Miao [5]. The present work is devoted to a detailed study of ac losses in Bi2212 round wires of different twist pitches. The main objectives are the characterization of filament decoupling at different pitch and operating conditions and identification of the effective coupling diameter and the

Partially supported by EuCARD-2 which is co-funded by the partners and the European Commission under Capacities 7th Framework Programme, Grant Agreement 312453. (Corresponding author: Yifeng Yang, e-mail: y.yang@soton.ac.uk.)

Y. Yang, J. Pelegrin, E.A Young and I. Faolorio are with The Institute of Cryogenics, Faculty of Engineering and the Environment, University of Southampton, Southampton SO17 1BJ, UK.

A. Ballarion and L Bottrura are with Technical Department, CERN, Geneva, Switzerland

H. Miao and Y. Huang are with Oxford Instruments Superconducting Technology, Carteret, New Jersey 07008, USA. email: hanping.miao@oxinst.com and Yibing.huang@oxinst.com time constant of the coupling current. 


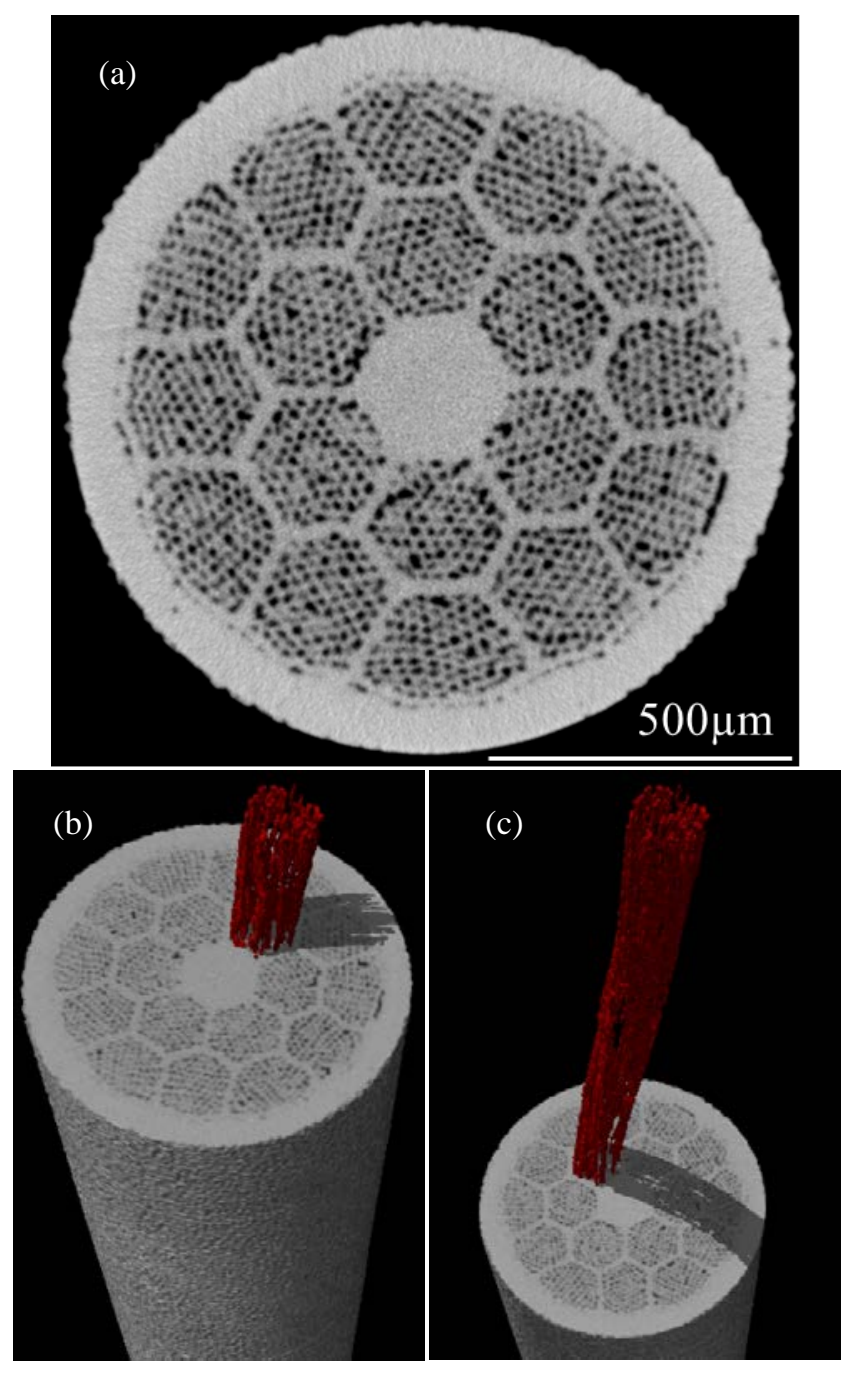

Fig. 1. (a) Cross-section of a Bi2212 round wire of $1.2 \mathrm{~mm}$ diameter with an 85x18 configuration. (b)-(c) X-ray tomographic images of the wire with the superconducting filaments in one of the sub-wires rendered volumetrically over a length of $L_{p} / 6$.

\section{EXPERIMENTAL}

\section{A. Bi2212 Round Wires}

Oxford Instruments Superconducting Technology (OST) has established a promising manufacturing route to produce silver sheathed Bi2212 round wires [5] with the powder-intube method. Multi-filamentary wires were produced by packing single-core wires in a hexagonal configuration and subsequently used for further restacking to yield conductors of a few thousand filaments. The wires could be twisted to a well-defined pitch while being drawn to the final diameter after the last restacking step.

The wires used in the present study had 1530 filaments in an $85 \times 18$ configuration of restacking 18 sub-wires of 85 filaments. A solid silver wire was placed at the centre in order to maintain the hexagonal symmetry of the final stacking and to provide further cryogenic stabilization. The wires had an outer diameter of $D=1.2 \mathrm{~mm}$ with twist pitches $L_{p}$ of $8 \mathrm{~mm}$, $12 \mathrm{~mm}$ and "infinity" (not twisted). The wire cross-section shown in Fig. 1(a) indicates an outer silver sheath of $0.1 \mathrm{~mm}$ thick, an inner diameter of $D_{i} \sim 1.0 \mathrm{~mm}$, and an average subwire diameter of $D_{s w}=0.25 \mathrm{~mm}$. In the X-ray tomographic images shown in Fig. 1(b)-(c), the superconducting filaments (volumes in red) in one of the sub-wires are rendered over a length of $\sim 1 / 6$ the twist pitch.

\section{B. AC Loss Measurements}

The present study focuses on the coupling current and hysteresis losses due to a sinusoidal external magnetic field [6], which was produced by an electromagnet with two symmetric air-gaps for the specimen and reference respectively. The electromagnet was able to provide fields up to $0.2 \mathrm{~T}$ in amplitude and $2 \mathrm{kHz}$ in frequency. The air gaps were able to accommodate samples up to $5 \mathrm{~mm}$ thickness, $15 \mathrm{~mm}$ wide, and $100 \mathrm{~mm}$ long. The length was a crucial requirement for investigating the (de)coupling of superconducting filaments. The present set-up allows the access to coupling current time constants in the millisecond regime.

The samples were cooled via a solid sapphire link to a GM cryo-cooler. The working temperature range is between $3 \mathrm{~K}$ and $100 \mathrm{~K}$.

The losses due to the ac magnetic field was measured using a simple saddle-like pick-up coil placed around the conductor. The symmetry of the dual air-gap design ensured an accurate phase measurement from the reference coil in the other airgap. The pick-up voltages were measured using a digital lockin amplifier to a noise floor $<10 \mathrm{nV}$ and at a phase angle resolution of $0.001^{\circ}$.

\section{Methodology and Limitations}

The use of a sinusoidal external ac field in this study was not because of a misguided anticipation of electrical power applications for the Bi2212 round wire. Instead it is designed for a detailed probe of the coupling current over a wide range of frequencies which are inaccessible in the standard field sweep magnetization measurement. The drawback of the ac field approach is the lack of dc magnetic field relevant to the intended operating conditions. This is due to the obvious difficulties of integrating an ac magnet suitable for a significant transverse sample length $(>\sim 100 \mathrm{~mm})$ necessary for coupling current studies. As the results presented here will show, such a limitation have been mostly mitigate by substituting magnetic field with temperature, which affects the conductor critical current in a similar manner. 
For each temperature $T$, the overall ac loss in an external field $B_{0} \cos (2 \pi f t)$ was measured by sweeping either the field amplitude $B_{0}$ at a given frequency or $f$ at a constant $B_{0}$.

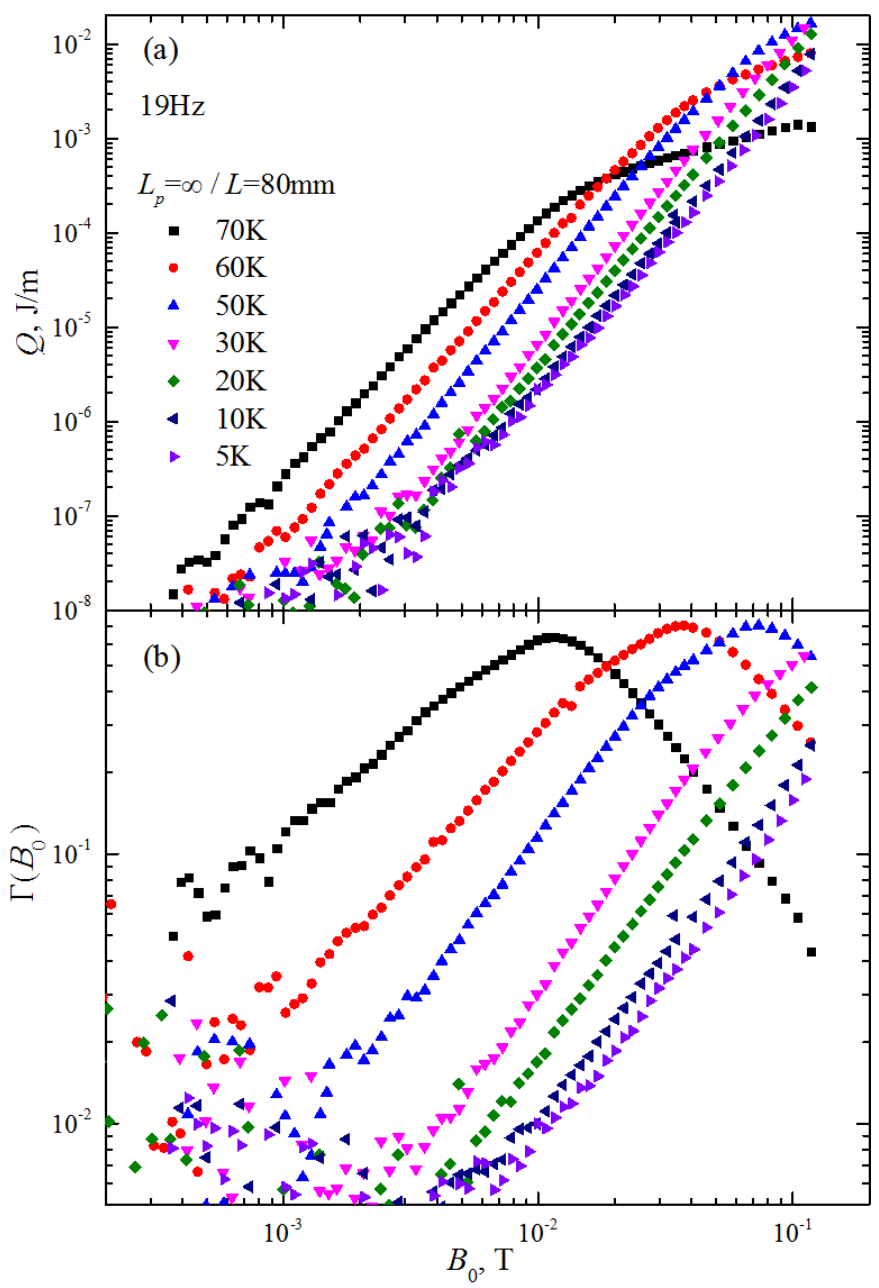

Fig. 2. (a) Losses per cycle and (b) the corresponding loss factor of a nontwisted $\mathrm{Bi} 2212$ wire as a function of ac field amplitude $B_{0}$ at different temperatures between $5 \mathrm{~K}$ and $70 \mathrm{~K}$.

\section{RESULTS AND DISCUSSION}

In simple conductors of a monolithic superconducting core or fully coupled superconducting filaments, the ac loss is dominated by the flux pinning induced magnetic hysteresis, which has been well understood. Fully filament coupling is expected when the conductor length exceeds the Rutherford uncoupling length criterion [7]:

$$
L_{c}=4 \sqrt{\frac{a \rho_{m} \lambda J_{C}}{\dot{B}}}
$$

where $a$ is the radius of either individual filaments or sub-wire bundles, $\rho_{\mathrm{m}}$ is the matrix resistivity, $\lambda$ is the superconductor fill factor ( $\lambda=1$ for coupling among individual filaments), $J_{\mathrm{C}}$ is the superconductor critical current. The average field sweep rate is approximately $\sqrt{ } 2 \pi f B_{0}$. For the $B i 2212$ wires in discussion here, the critical coupling length is just $\sim 75 \mathrm{~mm}$ for a high end $J_{\mathrm{C}}=5000 \mathrm{~A} / \mathrm{mm}^{2}$ filled at $25 \%(\lambda=0.25)$ within the 18 sub-wires of $125 \mu \mathrm{m}$ diameter in Ag of $\rho_{\mathrm{m}}=2 \times 10^{-10} \Omega \mathrm{m}$ in a small field of $B_{0}=1 \mathrm{mT}$ at $20 \mathrm{~Hz}$. It can also be concluded that (a) individual filaments $(5-10 \mu \mathrm{m})$ within a sub-wire would be fully coupled and (b) decoupling should be observed in wires with a twist pitch shorter than the coupling criterion.

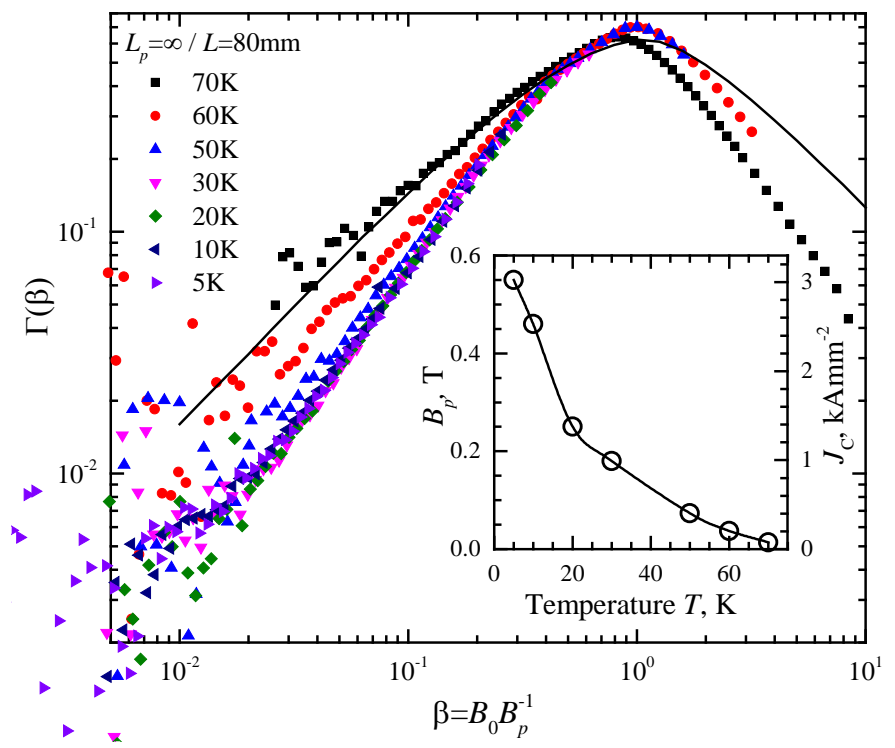

Fig. 3. Loss factor as a function of the normalized field $\beta=B_{0} / B_{p}$ for different temperatures between $5 \mathrm{~K}$ and $70 \mathrm{~K}$. The inset shows $B_{p}$ and the corresponding $J_{C}$ as a function of temperatures.

\section{A. Non-twisted Wires}

Fig. 2(a) shows the ac loss per cycle $Q$ at $19 \mathrm{~Hz}$, as a function of field amplitude $B_{0}$, of an $80 \mathrm{~mm}$ long non-twisted Bi2212 wire at different temperatures between $5 \mathrm{~K}$ and $70 \mathrm{~K}$. With the sample longer than the upper limit of Rutherford criterion, the wire is expected to behave as a monolithic superconducting cylinder with the 18 sub-wires fully coupled. The results shown in Fig. 2 indeed indicate a simple behavior of $Q \sim B_{0}{ }^{3+}$ for $B_{0}<B_{p}$ and $Q \sim B_{0}$ for $B_{0}>B_{p}$, where $B_{p}$ marks the fully penetration of the superconductor. The loss behavior is illustrated with further clarity in terms of loss factor $\Gamma=$ $\mu_{0} Q /\left(1 / 2 B_{0}{ }^{2} A_{Q}\right)$ which peaks at $B_{p}$ while increasing and decreasing with $\sim B_{0}{ }^{\sim \pm 1}$ respectively on either side. $A_{Q}$ is the loss producing cross-section area and the total wire area of $A_{w}$ $=\pi D^{2} / 4$ is used hereafter. The corresponding loss factor of the non-twisted wire in Fig. 2(b) confirms qualitatively the expected behavior for a monolithic superconductor. In addition, $B_{p}$ can be identified directly above $30 \mathrm{~K}$ with the full penetration within the maximum field magnitude of $0.2 \mathrm{~T}$.

The loss factor of a monolithic superconductor can be reduced to a single variable function of the normalized field $\beta$ $=B_{0} / B_{p}$, as shown by the solid line in Fig. 3 for a cylinder in transverse field. The non-twisted wire data in Fig. 2(b) is shown in terms of $\beta$ in Fig. 3. For temperatures $\sim 30 \mathrm{~K}, B_{p}$ is given by the peak position in Fig. 2(b). As the temperature is reduced, $\Gamma$ moves towards a steeper increase with $\beta$ than linearity for $\beta<1$ and converges to $\sim \beta^{1.3}$ for $T \leq 30 \mathrm{~K}$. It is therefore plausible, as shown in Fig. 3 , to determine $B_{p}$ for lower temperatures by scaling the corresponding data into a common curve. The full penetration field $B_{p}$, directly and indirectly obtained for temperatures above and below $30 \mathrm{~K}$ 
respectively, can be found in the inset of Fig. 3. Note that $B_{p}$ also leads explicitly to the critical current density $J_{C}\left(T, B_{p}\right)=$ $\lambda^{-1} \pi B_{p}(T) / 2 \mu_{0} D_{i}$, as indicated by the inset right axis. The upturn of $J_{C}$ below $20-30 \mathrm{~K}$ is consistent with the flux melting temperature of Bi2212 and $J_{C}(5 \mathrm{~K}, 0.5 \mathrm{~T}) \sim 3000 \mathrm{~A} / \mathrm{mm}^{2}$ is in line with that of the witness samples.

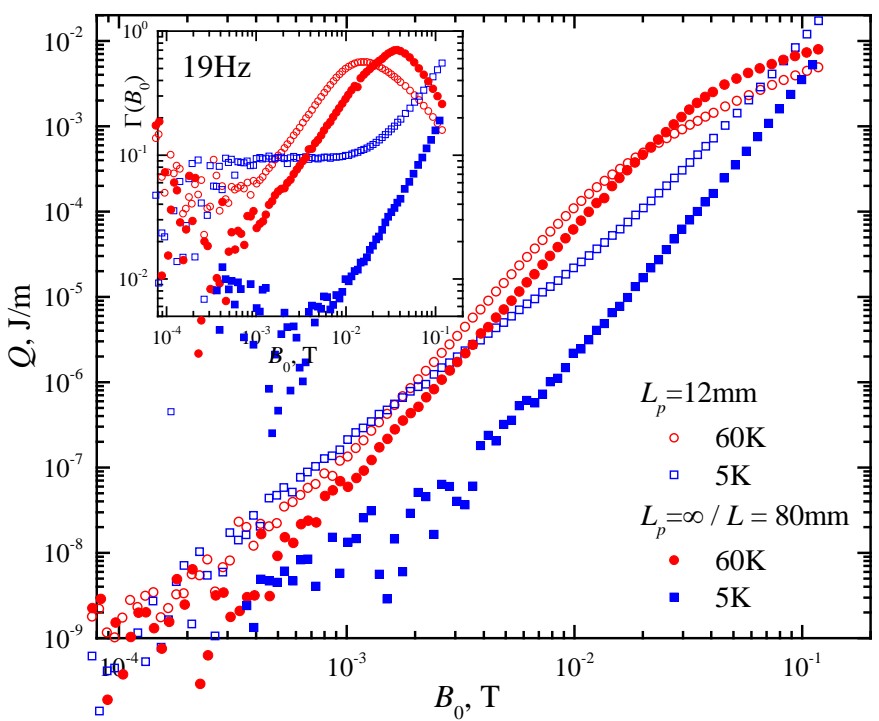

Fig. 4. The ac loss per cycle $5 \mathrm{~K}$ and $60 \mathrm{~K}$ (blue and red open symbols) of Bi2212 round wire with $L_{p}=12 \mathrm{~mm}$ is shown as a function of field amplitude $B_{0}$. The corresponding loss (solid symbols) of a non-twisted wire is also shown for comparison. The inset shows the loss factors of the same data set.

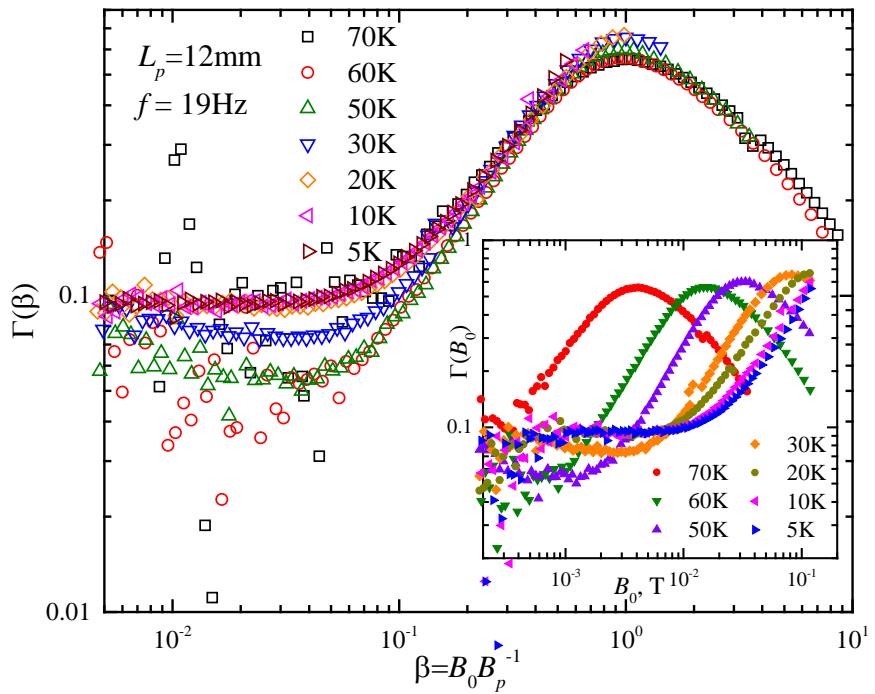

Fig. 5. Loss factor of a twisted wire with $L_{p}=12 \mathrm{~mm}$ at different temperatures as a function of field amplitude $B_{0}$ (inset) and as a function of the normalize field $\beta$.

\section{B. Twisted Wires}

Compared with the full coupling of sub-wires in the nontwisted wire, Fig. 4 shows a markedly different behavior for a wire twisted at a pitch of $12 \mathrm{~mm}$. At $60 \mathrm{~K}$, the twisted wire shows a reduced $B_{p}$, and a lower loss above $B_{p}$ than the nontwisted wire. It is the desired outcome of filament uncoupling by twisting. At field below $B_{p}$, uncoupling leads to a higher loss, which is inevitable but not an issue for high field applications where the imposed field always exceeds $B_{p}$ of the filaments/sub-wires. As shown in Fig. 5, the loss factor of the twisted wire of $L_{p}=12 \mathrm{~mm}$ at different temperatures (inset of Fig. 5) can be collapsed into a common one as $\Gamma(\beta)$ in a wide vicinity of $B_{p} \quad(0.1<\beta<10)$, confirming its origin of superconductor hysteresis.
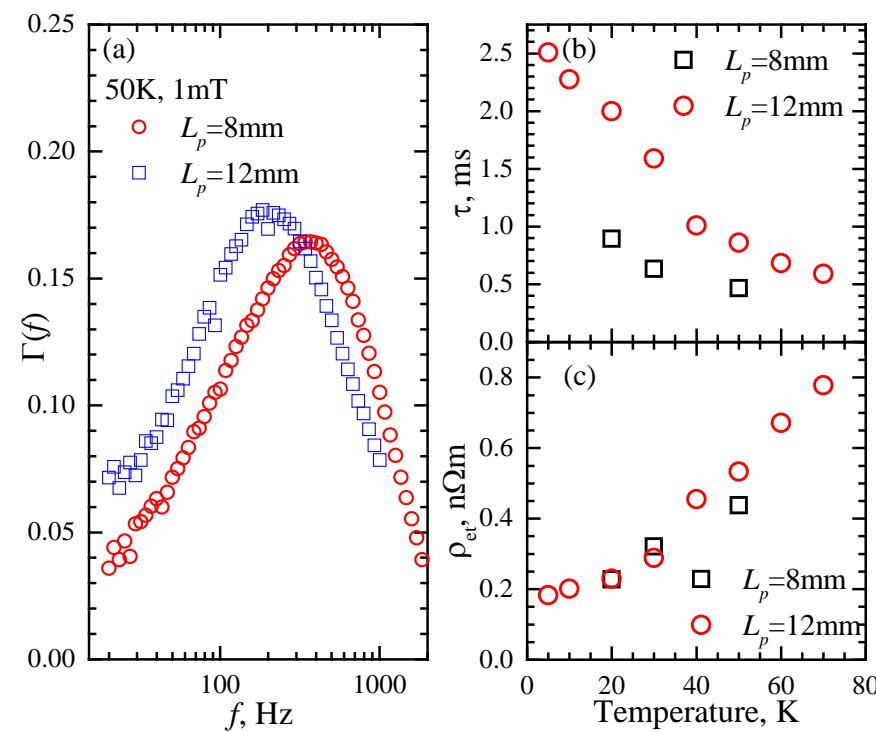

Fig. 6. For wires with different twist pitches of $8 \mathrm{~mm}$ and $12 \mathrm{~mm}$, (a) loss factor as a function of field frequency $f$, together with (b) time constant and (c) transverse resistivity at different temperatures.

\section{Coupling Current Losses and Time Constant}

At sufficiently small fields, coupling current loss becomes evident as $Q$ slows from $B_{0}{ }^{3+}$ towards $B_{0}{ }^{2}$, which corresponds to a constant loss factor as found in the Fig. 4 inset and in Fig. 5. The dominance of the coupling current loss extends to higher field at lower temperatures with increasing $B_{p}$. Note that the coupling current loss does not scale with $\beta$ for different temperatures. Instead, the losses reduce with increasing temperature at $19 \mathrm{~Hz}$.

The loss factor of coupling current among the twisted filaments is given by [8]:

$$
\begin{aligned}
& \Gamma_{C P}(z)=\lambda_{f} \frac{\pi z}{z^{2}+1} \text { and } z=2 \pi f \tau_{f} \\
& \tau_{f}=\lambda_{f} \frac{\mu_{0}}{2 \rho_{e t}}\left(\frac{L}{2 \pi}\right)^{2}
\end{aligned}
$$

where $\lambda_{f}<1$ is the effective internal flux fill factor which takes into account the diamagnetic contribution from the filaments, and $\tau_{f}$ is the effective time constant of the coupling current. As a function of frequency, $\Gamma_{\mathrm{CP}}$ reaches a peak value of $1 / 2 \pi \lambda_{f}$ at $2 \pi f \tau_{f}=1$. Fig. $6(\mathrm{a})$ indicates $\Gamma_{\mathrm{CP}, \max } \sim 0.17$ hence $\lambda_{f} \sim 0.1$. The peak frequencies are $390 \mathrm{~Hz}$ and $185 \mathrm{~Hz}$ for $L_{p}=8 \mathrm{~mm}$ and $12 \mathrm{~mm}$ respectively. Note that the ratio of the peak frequency is close to the expected 2.25 by the $L_{p}^{2}$ dependence.

It is encouraging that the time constant $\tau_{f}$, shown in Fig. 6(b) for the two wires of different pitch, is in the millisecond regime. The increase of $\tau_{f}$, with reducing temperature is the result of lower matrix resistivity. Equation (2) highlights the low flux fill factor as the ingredient for the short time constant, especially for a transverse resistivity $\rho_{\mathrm{et}}$ (Fig. 6(c)) almost as low as that of pure Ag at at a Residual 
Resistance Ratio RRR=100.
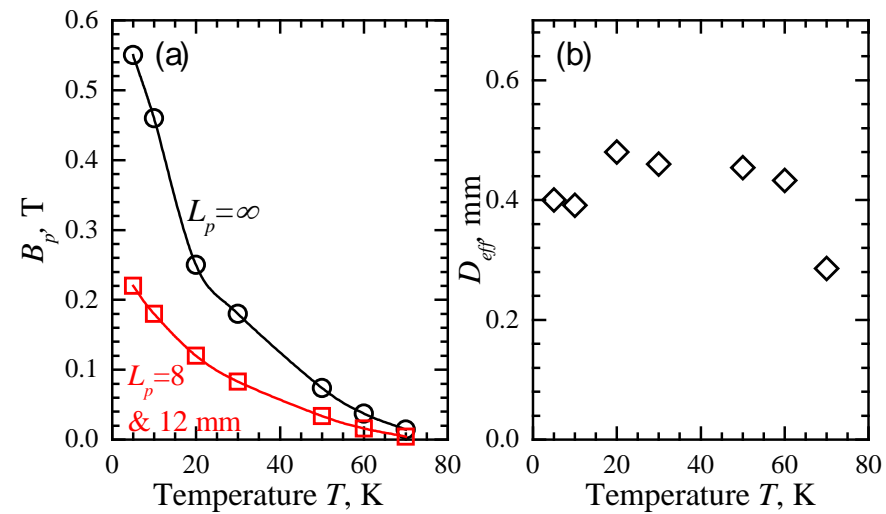

Fig. 7. (a) Full penetration field of twisted ( $B_{p, L p}$, with $L_{p}=8 \mathrm{~mm}$ and $12 \mathrm{~mm}$ ) and non-twisted $\left(B_{p, \infty}\right)$ at different temperatures and (b) deduced effective diameter of coupling.

\section{Effective Diameter of Decoupling}

Similar to the non-twisted wire, the full penetration field $B_{p}$ of twisted wires can be determined either directly at $T \geq 20 \mathrm{~K}$ using the peak position of $\Gamma\left(B_{0}\right)$ or indirectly by achieving a common $\Gamma(\beta)$ scaling. The results are shown in Fig. 7(a) together with $B_{p}(T)$ of the non-twisted wire. Assuming no $J_{C}$ degradation due to twisting, the effective diameter of coupling is $D_{\text {eff }}=B_{p, L p} / B_{p, \infty} \sim 0.4 \mathrm{~mm} \sim 1.6 D_{s w}$, as shown in Fig. 7b. Such an effective diameter of coupling seems to suggest a partial of coupling of neighbouring sub-wires.

\section{CONCLUSION}

AC loss measurements at a wide range of temperatures ( $5 \mathrm{~K}$ - $70 \mathrm{~K}$ ) with sweeping the amplitude and frequency of ac sinusoidal field have revealed rich details of the hysteresis and coupling current losses. The twisted conductors showed classical behavior of uncoupling by filament twisting, although some partial coupling among adjacent sub-wires still existed with the effective coupling diameter of $60 \%$ larger than the sub-wire diameter.

The coupling current loss was rather low, primarily due to a small flux fill factor $\lambda_{f}$ of just $10 \%$. The same mechanism also led to a promising short time constant for the coupling current, even with the transverse resistive matrix behaving like pure silver. Hence a low contact resistance between the filaments and the matrix was also implied. It should be noted that the small flux fill factor could change in high external field and with wire architecture. It is an important aspect for future studies.

The standard expressions for the critical coupling length and the time constant of the coupling current seemed to hold well and the simple sum of coupling current and hysteresis losses appeared to work satisfactorily. However the penetration loss was not observed. It might be the result of partial coupling among the adjacent sub-wires and should be an important topic for further investigation due to its importance in the fully penetrated regime.

\section{REFERENCES}

[1] C. Friend, H. Miao, Y. Huang, Z. Melhem, F. Domptail, M. Meinesz, S. Hong, Y. Yang, and E. Young, "The development of high field magnets utilizing Bi-2212/Ag wind \& react inset coils,” IEEE Trans. Appl. Supercond., vol. 20, no. 3, pp. 583-586, Jun. 2010.

[2] Y. Yang, E.A. Young, I. Falorio, W.O.S. Bailey, C.M. Friend, A. Twin, S.P.G. Chappell, "Quench characteristics of Bi2212 solenoid insert coils in background field up to 20 T," IEEE Trans. Appl. Supercond. 21, no. 3, pp. 2432-2435, Jun 2011.

[3] D.C. Larbalestier, J. Jiang, U.P. Trociewitz, F. Kametani, C. Scheuerlein, M. Dalban-Canassy, M. Matras, P. Chen, N.C. Craig, P.J. Lee, E.E. Hellstrom, "Isotropic Round-Wire Multifilament Cuprate Superconductor for Generation of Magnetic Fields above 30 T”, Nature Materials, 13 375-381, 2014.

[4] J. Jiang, W. L. Starch, M. Hannion, F. Kametani, U. P. Trociewitz, E. E. Hellstrom, and D. C. Larbalestier, "Doubled critical current density in Bi-2212 round wires by reduction of the residual bubble density," Supercond. Sci. and Technol., vol. 24, no. 8, p. 082001, 2011.

[5] Y. Huang, H. Miao, S. Hong, and J. A. Parrell, "Bi-2212 round wire development for high field applications,” IEEE Trans. Appl. Supercond. vol. 24, 06400205, 2014.

[6] E.A. Young, M. Bianchetti, G. Grasso, Y. Yang, "Characteristics of AC Loss in Multifilamentary MgB2 Tapes”, IEEE Trans. Appl. Supercond., vol. 17, no. 2, pp. 2945 - 2948, Jul. 2007

[7] P. F. Smith et al, in "Proceedings of Summer Study on Superconducting Devices and Accelerators”, Brookhaven, 1968, edited by A. G. Prodell and H. Hahn. (Brookhaven National Laboratories, Brookhaven, N.Y.) BNL 50155 (C-55), p. 913, 1969

[8] G.H. Morgan, "Theoretical Behavior of Twisted Multicore Superconducting Wire in a Time-Varying Uniform Magnetic Field”, $J$. Appl. Phys. Vol. 41, pp. 3673-3679, 1970. 